\title{
The use of cardiac magnetic resonance imaging (CMRI) for adult congenital heart disease patients: qualitative comparative review
}

\author{
Author: Ahmed Alsunbuli ${ }^{\mathrm{A}}$
}

\section{Introduction}

Grown-up congenital heart diseases (GUCH) are a new field in cardiology due to historically high childhood mortality rates. ${ }^{1}$ Compared with 60 years ago, pre-adulthood mortality rates have dropped from $90 \%$ to $10 \%$. Once they become an adult, patients with GUCH need follow-up and monitoring of their conditions. ${ }^{2}$ Many of them need lifelong monitoring, and thus the need for a reliable imaging modality emerges.

\section{Materials and methods}

This study compares different imaging modalities based on their innate characteristics plotted against a virtual ideal test, as well as the different societies' guidelines, utilising a qualitative approach to the comparison. A systematic search for evidence was conducted looking into resources such as PUBMED, EMBASE, ScienceDirect, CINAHL, NICE, ESC, ACC/AHA using Boolean operators with phrases like: 'CMRI', 'GUCH', 'ACHD', 'diagnostic modalities', 'imaging techniques'. After primary selection of included resources, the studies were analysed for inclusion in the body of evidence.

\section{Results and discussion}

Cardiac magnetic resonance imaging (CMRI) has no radiation risk but offers lower resolution than computed tomography (CT); it is more time consuming and therefore, more demanding for clinicians and patients. ${ }^{3}$ It is less operator dependent than echocardiography which allows for detection of minor changes in serial follow-up assessments mainly for left ventricular volume and function. CMRI is also applicable in pregnancy. On the downside, it is contraindicated in the presence of certain pacemakers, and it cannot be utilised intra-procedurally. CMRI is also prone to artefacts that can be identified through chest $\mathrm{X}$-ray, such as retrocardiac surgical needle in one case. ${ }^{4}$

CMRI is uniquely indicated in right ventricular volume and ejection fraction assessment, as well as for abnormalities in the great vessels and for pulmonary artery conduits. ${ }^{5} \mathrm{CMRI}$ is rarely done as a first-line test. Usually, it is utilised to answer

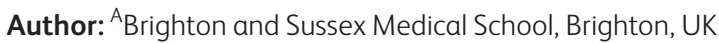

Table 1. Point scoring of different imaging modalities in grown-up congenital heart diseases. The values are based on the research conducted in the study.

\begin{tabular}{l|l|l|l} 
& MRI & TIE & CT \\
\hline Extra-cardiac data collection & +++ & + +- & +++ \\
\hline detection of smaller changes (i.e. LV size, ...) & +++ & ++ & +++ \\
\hline no radiation exposure & +++ & +++ & - \\
\hline non-invasive & +++ & +++ & +++ \\
\hline operator independent & +++ & + & +++ \\
\hline special circumstances: pregnancy & +++ & +++ & - \\
\hline Total & $18 / 18$ & $12.5 / 18$ & $12 / 18$ \\
\hline
\end{tabular}

$\mathrm{CT}=$ computed tomography; $\mathrm{LV}=$ left ventricular; $\mathrm{MRI}=$ magnetic resonance imaging; TTE = transthoracic echocardiography.

predetermined morphological and haemodynamic questions of already configured anatomy. ${ }^{4}$ Furthermore, the latest developments of 4D-CMRI carry the potential of identifying risk profiles and treating patients before developing clinical manifestations by recognising areas of wall strain and flow patterns. $^{6}$

\section{Conclusion}

No single test is perfect for all patients with GUCH all of the time, but CMRI proves to be near perfect when it comes to serial follow-up and definitive diagnosis (Tables 1 and 2). It falls short when faced with ferromagnetic foreign bodies, intraoperative imaging, emergencies, suboptimal patient

Table 2. Overall numerical qualitative value for different imaging modalities in grown-up congenital heart diseases

\begin{tabular}{|c|c|c|c|c|}
\hline Imaging modality & $\begin{array}{l}\text { Marcotte } \\
\text { et } a l^{7}\end{array}$ & $\begin{array}{l}\mathrm{ACC} / \\
\mathrm{AHA}^{8}\end{array}$ & $\begin{array}{l}\text { This } \\
\text { study }\end{array}$ & Total \\
\hline MRI & 33 & 14 & 18 & 65 \\
\hline Echocardiography & 26 & 9 & 12.5 & 47.5 \\
\hline Cardiac CT & 23 & 12 & 12 & 47 \\
\hline
\end{tabular}


cooperation, or with vegetations and small thrombi where cardiac CT is superior; and it is more expensive (than echocardiography) to be used for initial patient assessment.

\section{Conflicts of interest}

None declared.

\section{References}

1 Baumgartner H. An important attempt to improve the outcome of congenital heart disease in Europe. Eur Heart J 2014;35:674.

2 Pandya B, Cullen S, Walker F. Congenital heart disease in adults. BMJ 2016;354:i3905.

3 Fratz S, Chung T, Greil GF et al. Guidelines and protocols for cardiovascular magnetic resonance in children and adults with congenital heart disease: SCMR expert consensus group on congenital heart disease. J Cardiovasc Magn R 2013;15:51.

4 Fratz S, Hess J, Schuhbaeck A et al. Routine clinical cardiovascular magnetic resonance in paediatric and adult congenital heart disease: patients, protocols, questions asked and contributions made. J Cardiovasc Magn R 2008;10:46.

5 Baumgartner H, Bonhoeffer P, De Groot NMS et al. ESC guidelines for the management of grown-up congenital heart disease (new version 2010) the task force on the management of grown-up congenital heart disease of the European Society of Cardiology (ESC). Eur Heart J 2010;31:2915-57.

6 François CJ, Srinivasan S, Schiebler ML et al. 4D cardiovascular magnetic resonance velocity mapping of alterations of right heart flow patterns and main pulmonary artery hemodynamics in tetralogy of Fallot. J Cardiovasc Magn R 2012;14:16.

7 Marcotte F, Poirier N, Pressacco J et al. Evaluation of adult congenital heart disease by cardiac magnetic resonance imaging. Congenit Heart Dis 2009;4:216-30.

8 Stout KK, Daniels CJ, Aboulhosn JA et al. 2018 AHA/ACC guideline for the management of adults with congenital heart disease: executive summary: a report of the AHA/ ACC Task Force on Clinical Practice Guidelines. Circulation 2019;139:e637-97. 